\title{
Competition between global feedback and diffusion in coupled Belousov-Zhabotinsky oscillators
}

\author{
Kota Ohno, Toshiyuki Ogawa, ${ }^{*}$ and Nobuhiko J. Suematsu \\ Graduate School of Advanced Mathematical Science, Meiji University, Nakano, Tokyo 164-8525, Japan \\ and Meiji Institute for Advanced Study of Mathematical Sciences, Meiji University, Nakano, Tokyo 164-8525, Japan
}

(Received 5 September 2018; revised manuscript received 22 October 2018; published 10 January 2019)

\begin{abstract}
The Belousov-Zhabotinsky (BZ) reaction is a famous experimental model for chemical oscillatory reaction and pattern formation. We herein study a diffusive coupled system of two oscillators with global feedback using the photosensitive BZ reaction both experimentally and theoretically. The coupled oscillator showed in-phase and antiphase oscillations depending on the strength of diffusive coupling and light feedback. Moreover, we analyzed our model to locate the bifurcational origin and found the reconnection of the bifurcation branches for antiphase oscillation, which was induced by the competition between global feedback and the diffusion effect.
\end{abstract}

DOI: 10.1103/PhysRevE.99.012208

\section{INTRODUCTION}

Pattern dynamics has been drawing attention in many research fields such as chemical reaction, thermal convection, ecology, and physiology. Further, it has been explained using mathematical models [1].

The Belousov-Zhabotinsky (BZ) reaction is a famous experimental model that produces both excitable and oscillatory patterns. One can also control the pattern dynamics of the BZ reaction with $\mathrm{Ru}(\mathrm{bpy})_{3}^{2+}$ catalyst through light illumination [2]. Oscillations and wave propagations in the photosensitive BZ reaction are inhibited by light stimulation because photochemical reaction produces the inhibitor $\mathrm{Br}^{-}[3,4]$. Using this property, a light feedback BZ system that can be used in excitable situations has been reported. In fact, Mihaliuk et al. reported that they localized the traveling wave and controlled its movements [5-7]. Nishi et al. reported that they eliminated the propagation wave [8]. In the above reports, they introduced theoretical studies using the Oregonator model.

Meanwhile, several studies have been conducted for the light feedback BZ system in oscillatory conditions. Petrov et al. reported that standing waves were observed via periodic light stimulation to the photosensitive $\mathrm{BZ}$ reaction [9]. They found that the wavelength of the standing wave patterns depends on the frequencies of the light stimulation. However, this might not indicate the intrinsic behaviors of the system because Petrov's system is a forced oscillatory control system. Therefore, we would like to realize oscillatory patterns using feedback control instead. It is noteworthy that the research by Vanag et al. pertains to this area [10,11]. They observed standing waves using a global feedback system for the photosensitive BZ reaction. Moreover, they introduced a mathematical model to study spatiotemporal patterns in the global feedback system of the BZ reaction [11]. In addition, coupled BZ oscillators with inhibitory diffusion and negative global feedback has been recently reported [12]. They studied dynamics of a one-dimensional (1D) array and observed

\footnotetext{
*togw@meiji.ac.jp
}

several kinds of oscillatory cluster patterns with changing feedback strength in experiments and numerical simulations. Kashima et al. introduced a theoretical study of feedback control for Turing patterns or oscillatory patterns produced by wave instability using the idea of semipassivity $[13,14]$.

It has been reported that many varieties of oscillatory cluster patterns appear depending on the initial condition if we observe the $2 \mathrm{D}$ problem with global feedback [10,11]. They observed oscillatory cluster patterns both in the experiment and in a modified BZ model. We can also observe a similar behavior using the three-component Oregonator model in the $1 \mathrm{D}$ or $2 \mathrm{D}$ region $\Omega$ as follows [10,11]:

$$
\begin{aligned}
\frac{\partial u}{\partial t} & =D_{u} \Delta u+\frac{1}{\varepsilon}[q v-u v+u(1-u)], \\
\frac{\partial v}{\partial t} & =D_{v} \Delta v+\frac{1}{\delta}(-q v-u v+f w+s \phi), \\
\frac{\partial w}{\partial t} & =D_{w} \Delta w+u-w, \\
\phi & =\frac{1}{|\Omega|} \int_{\Omega} w d x .
\end{aligned}
$$

In fact, antiphase-like oscillations such as standing oscillation patterns or localized standing patterns are observed.

We herein propose a simple situation to clarify the type of mechanism that causes such antiphase-like behaviors. More precisely, we study the behaviors of two BZ oscillators coupled via diffusion, instead of $2 \mathrm{D}$ domains, with negative global feedback effect as well. This can be considered to be the simplest oscillatory cluster pattern. One can imagine that in-phase oscillation is observed with diffusive coupling only and no negative global feedback [15-19]. On the contrary, antiphase oscillation may be observable with negative global feedback only and with no diffusive coupling. Therefore, the behavior of coupled BZ oscillators under the competition between global feedback and diffusion effects is an interesting research topic.

First, we study a mathematical model, i.e., the threevariable Oregonator model modified for the photosensitive 
BZ reaction, and observe two different types of oscillations: in-phase and antiphase oscillations. (See Fig. 1 in detail.) The period of antiphase oscillations increases by increasing feedback strength. We analyzed our model to locate the bifur-
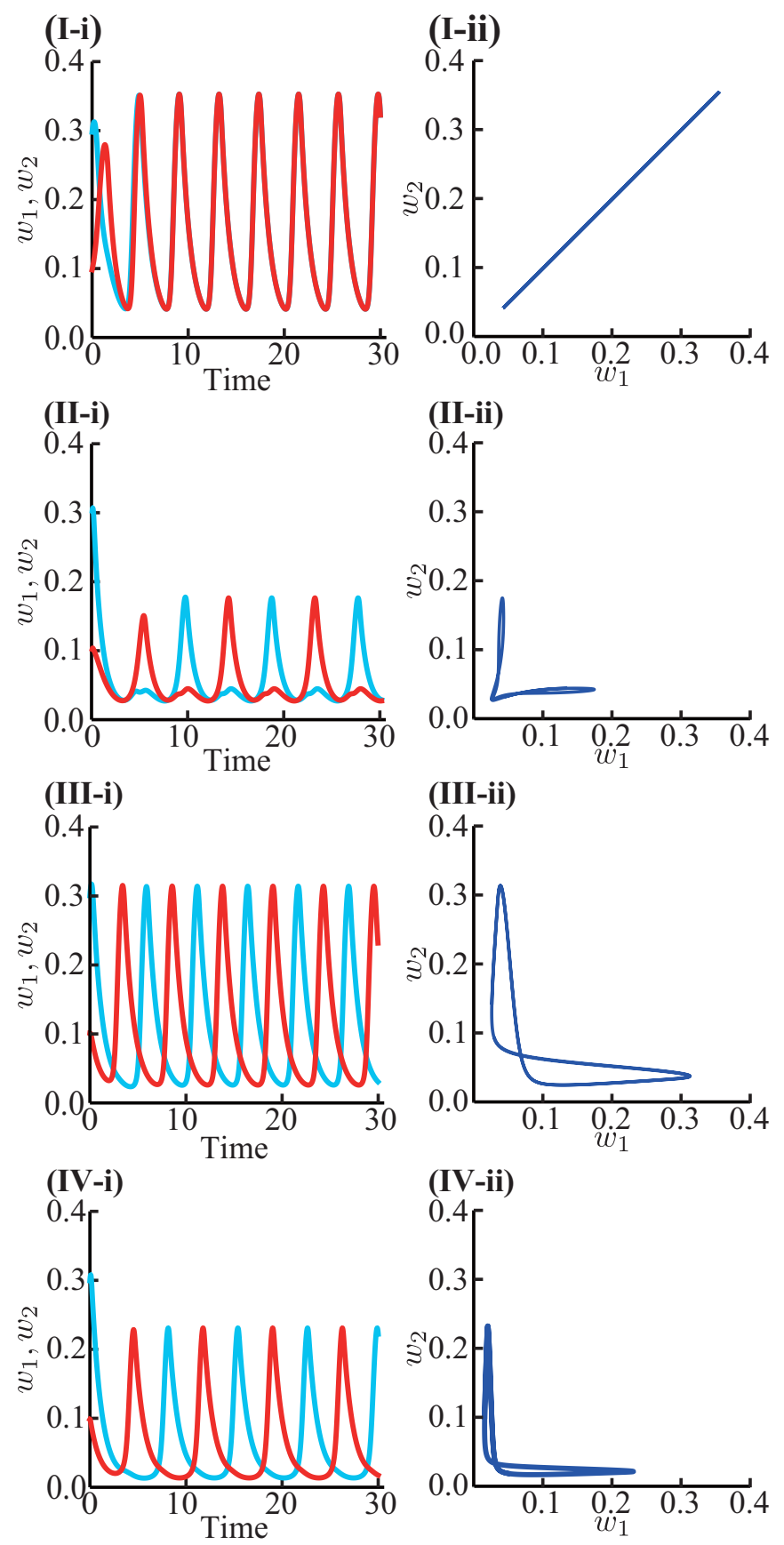

FIG. 1. (i) Time profiles and (ii) phase plane plots of $w_{i}$ for stable periodic solutions. (I) In-phase oscillation. [(II), (III), and (IV)] Antiphase oscillation. Parameters are (I) $D=0.3, s=0.0$; (II) $D=0.3, s=1.0$; (III) $D=0.0, s=0.3$; and (IV) $D=0.0, s=$ 1.0. In the case of $D=0.3$, we can observe the transition from in-phase (I) to antiphase oscillation (II) by increasing $s$. Meanwhile, without diffusion coupling, antiphase oscillation gradually changes its period as $s$ becomes larger (III and IV). We used fourth-order Runge-Kutta method for these numerical simulations. The time step size is $d t=0.0002$. cational origins of antiphase oscillations by varying the global feedback strength as a bifurcation parameter. In fact, when the diffusive coupling is sufficiently small, antiphase oscillation is stable for any feedback strength. Meanwhile, if the diffusive coupling is reasonably large, then the branch of the stable antiphase oscillation originates from the period-doubling bifurcation point along the branch of the in-phase oscillation. Moreover, the reconnection of two different branches is observed by the two-parameter search. Therefore, the branch of antiphase oscillations may have different bifurcational origins depending on the diffusion effects.

Next, we experimentally observed synchronization phenomena and measured the periods of oscillation. Our experimental system was composed of two flow reactors coupled by a pump and light feedback system. A flow reactor can keep the concentration of reactants constant. Therefore, it is suitable for observing the oscillation for a long time. In addition, the pump system is appropriate for coupling the flow reactors and controlling the strength of coupling [15]. Moreover, the experimental results well agree with the bifurcation diagram obtained by our model.

\section{MATHEMATICAL MODEL}

\section{A. MODEL}

We consider a coupled system of two oscillators that are described by the Oregonator model [20,21]. The oscillators are connected by two types of coupling terms-diffusion and light feedback,

$$
\begin{aligned}
\frac{d u_{i}}{d t} & =\frac{1}{\varepsilon}\left[q v_{i}-u_{i} v_{i}+u_{i}\left(1-u_{i}\right)\right]+D\left(u_{j}-u_{i}\right), \\
\frac{d v_{i}}{d t} & =\frac{1}{\delta}\left(-q v_{i}-u_{i} v_{i}+f w_{i}+s \phi\right)+D\left(v_{j}-v_{i}\right), \\
\frac{d w_{i}}{d t} & =u_{i}-w_{i}+D\left(w_{j}-w_{i}\right), \\
\phi & =\frac{w_{1}+w_{2}}{2} . \quad \quad(i, j=1,2, i \neq j)
\end{aligned}
$$

Here $\mathbf{u}_{i}(t)=\left(u_{i}(t), v_{i}(t), w_{i}(t)\right)(t>0)$ are dimensionless variables that correspond to the concentrations of the activator $\mathrm{HBrO}_{2}$, inhibitor $\mathrm{Br}^{-}$, and oxidized catalyst $\mathrm{Ru}(\mathrm{bpy})_{3}^{3+}$, respectively, for the oscillator $i$. We use the typical parameter set for the limit cycle oscillation: $\varepsilon=0.1, \delta=0.001, f=$ 1.0 , and $q=0.01$. The diffusion coefficients for $u, v, w$ are assumed to be the same positive constant $D$. We describe the light feedback effect by adding $s \phi$ to the equation for $v$, where $\phi$ is the average concentration of $w_{1}$ and $w_{2}$ and $s$ is a positive constant corresponding to the feedback strength $[3,4,8]$. We consider $s$ and $D$ as bifurcation parameters.

\section{B. Numerical results}

Two different types of stable oscillations are numerically observed, as shown in Fig. 1: in-phase and antiphase. Suppose $\left(\mathbf{u}_{1}(t), \mathbf{u}_{2}(t)\right)$ is the periodic solution of Eq. (2) with period $T$. We call $\left(\mathbf{u}_{1}(t), \mathbf{u}_{2}(t)\right)$ in-phase oscillation if $\left(\mathbf{u}_{1}(t), \mathbf{u}_{2}(t)\right)$ $\equiv\left(\mathbf{u}_{2}(t), \mathbf{u}_{1}(t)\right)$ holds. On the other hand, we call $\left(\mathbf{u}_{1}(t)\right.$, $\left.\mathbf{u}_{2}(t)\right)$ antiphase oscillation if $\left(\mathbf{u}_{1}(t), \mathbf{u}_{2}(t)\right) \equiv\left(\mathbf{u}_{2}(t+T / 2)\right.$, $\left.\mathbf{u}_{1}(t+T / 2)\right)$ holds. In fact, in-phase oscillations are observed 
under the nonfeedback condition $(s=0.0)$, as shown in Fig. $1(\mathrm{I}-\mathrm{i})$ and 1(I-ii), when diffusion coupling exists. On the other hand, antiphase are observed under the feedback condition [Figs. 1(II-i), 1(II-ii), 1(III-i), 1(III-ii), 1(IV-i), and 1(IV-ii)]. In the case of $D=0.3$, we can observe the transition from in-phase [Figs. 1(I-i) and 1(I-ii)] to antiphase oscillation [Figs. 1(II-i) and 1(II-ii)]. Meanwhile, without diffusion coupling, antiphase oscillations are observed, and its period gradually changes with the increase in $s$ [compare Figs. 1(III-i) and 1(IV-i)]. Notice that the profile and the period of $\mathbf{u}_{i}(t)$ for (a-i)
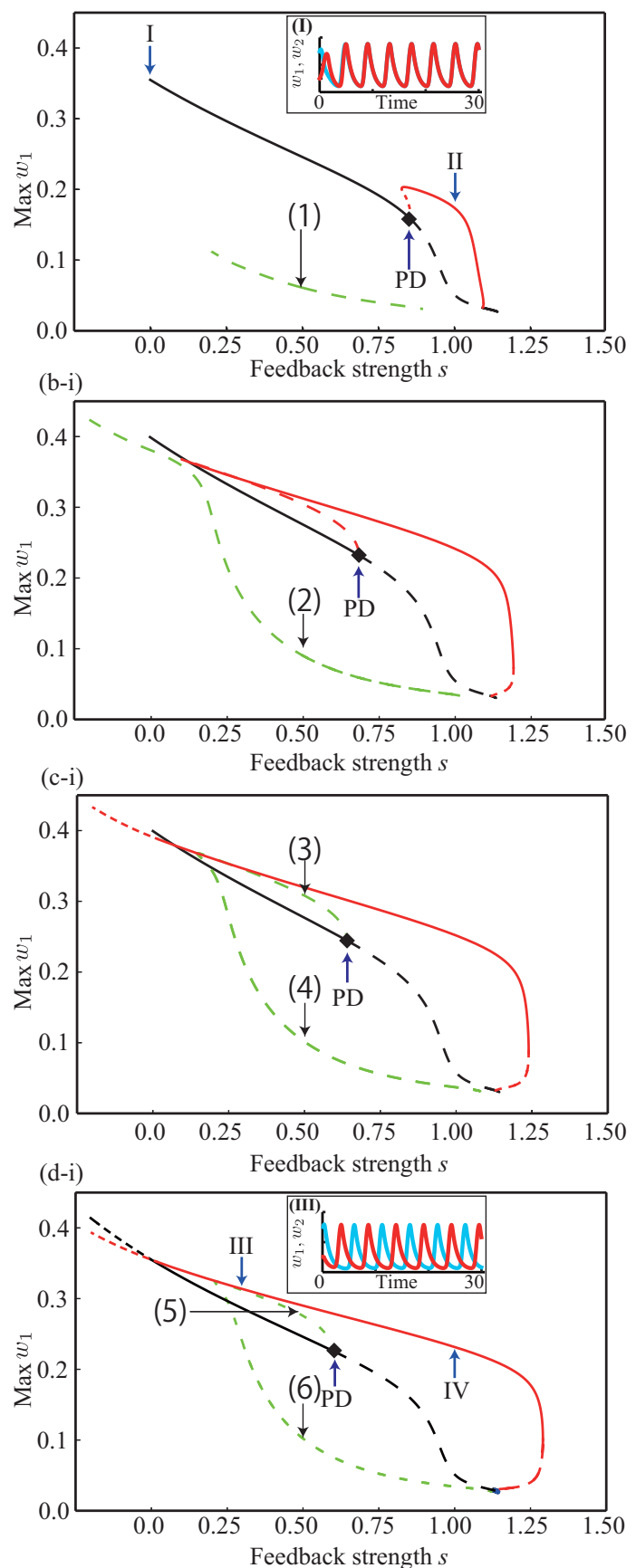

(a-ii)
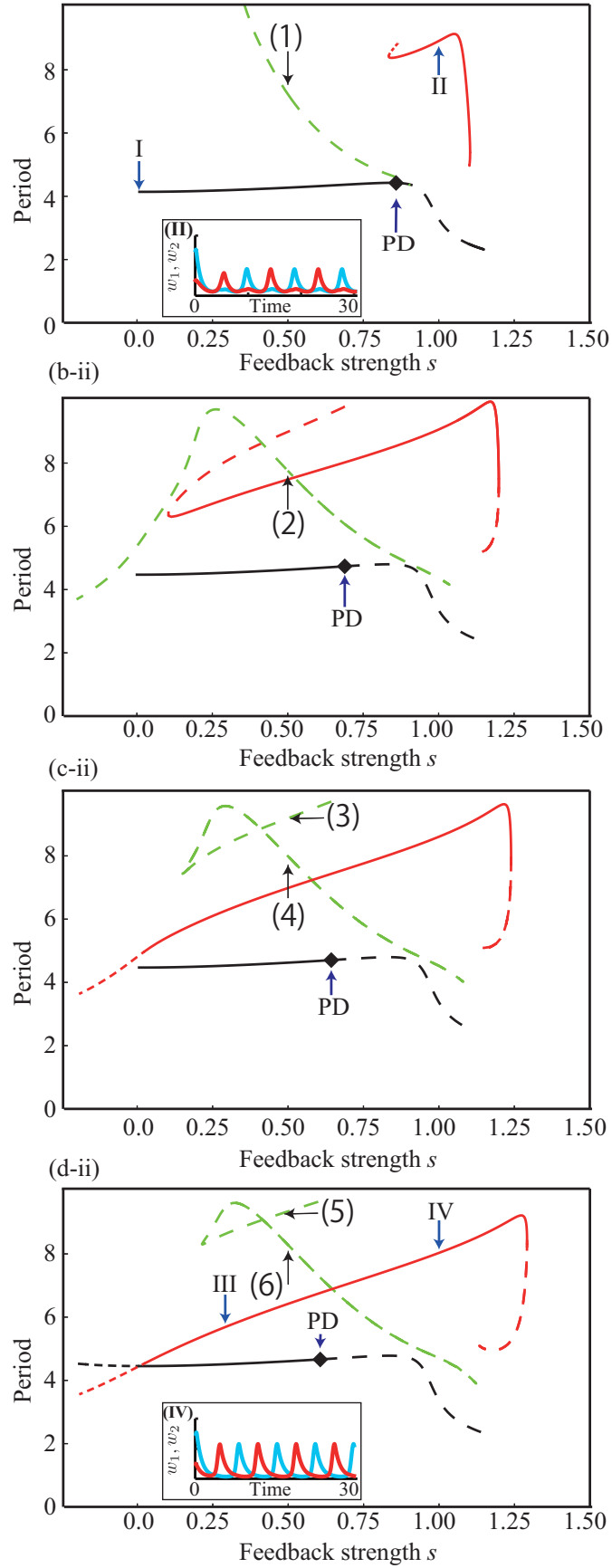

FIG. 2. Bifurcation diagrams for Eq. (2) with respect to parameter $s$. Diffusion constant $D$ is (a) 0.3 , (b) 0.1 , (c) 0.05 , and (d) 0.0 . We describe (i) the maximum value of $w_{1}$ and (ii) the period of the periodic orbits. There are three branches of periodic solutions. One is the branch of in-phase oscillations (black). The solutions on the other two branches [red (dark gray) and green (light gray)] are antiphase oscillations. The solid curves represent the branch of stable solution, while the broken curves represent the unstable branch. "PD" indicates the period-doubling bifurcation point. Insets (I)-(IV) show the time profiles of $w_{i}$, which are the same as Fig. 1. The profiles for (1)-(6) are shown in Fig. 4. These oscillations are antiphase oscillations. 


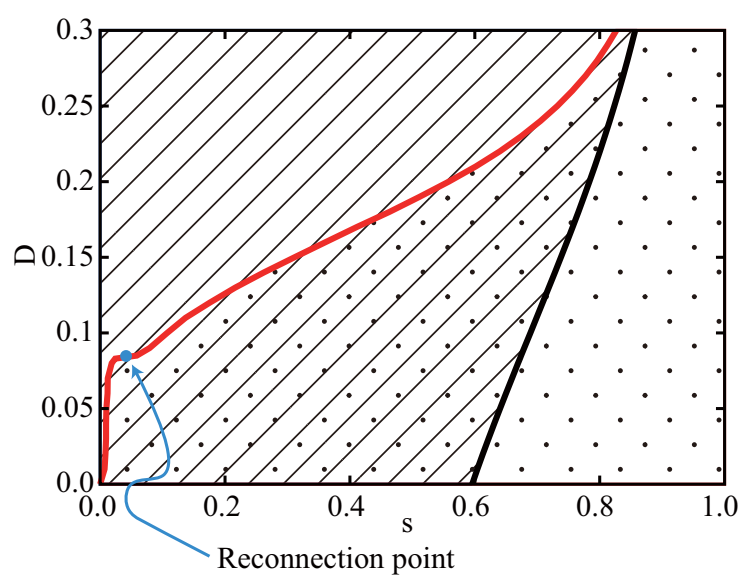

FIG. 3. Phase diagram on $(s, D)$ space. In-phase oscillations are stable in the hatched area. Antiphase oscillations are stable in the dotted area. The black line corresponds to the period-doubling bifurcation point on the branch of in-phase oscillations. The red (dark gray) line corresponds to the saddle-node bifurcation point on the branch of antiphase oscillations when $D$ is larger than the reconnection point. On the contrary, when $D$ is less than the reconnection point, it corresponds to a critical point for stability of antiphase oscillations. Since this critical point stays close to $s=0$, the antiphase oscillation is stable for almost all the values of $s$. Moreover, this critical point corresponds to a stationary bifurcation in the sense of Poincaré map along the periodic orbit.

antiphase oscillations in [Figs. 1(II-i) and 1(IV-i)] are quite different from those for in-phase oscillations. Therefore, we have extended the definition of antiphase oscillation.

\section{Bifurcation analysis}

We performed bifurcation analysis using the software package AUTO [22] to understand the origin of the antiphase oscillations. We demonstrate the bifurcation diagrams obtained from a two-parameter search by $s$ and $D$ (Fig. 2). We detect these diagrams from in-phase oscillation at $(s, D)=$ $(0.0,0.3)$ as a starting point.

It turns out that in-phase oscillations exist stably for a wide range of parameter $s$, independent of $D$. The critical point for the stability on the branch of the in-phase oscillation corresponds to the period-doubling bifurcation. The branch starting from the period-doubling point corresponds to unstable oscillations when $D$ is small [Figs. 2(c-i), 2(c-ii), 2(d-i), and 2(d-ii)]. Meanwhile, when $D$ is large, it continues as the stable branch of the antiphase oscillations [Figs. 2(a-i), 2(a-ii), 2(b-i), and 2(b-ii)]. When $D$ is sufficiently small, a long stable branch of antiphase oscillations appears [Figs. 2(c-i), 2(c-ii), 2(d-i), and 2(d-ii)]. The above is expressed in a phase diagram, as shown in Fig. 3. Furthermore, the green (light gray) branches are isolated in the bifurcation diagram of $s$. However, these are connected by two-parameter search. Therefore, two-parameter continuation is available. It is noted that we show time profiles for the green (light gray) branches as shown in [Figs. 4(1-6)].

By carefully changing $D=0.05-0.1$, we can see the reconnection of the two branches: the long stable branch of the antiphase [red (dark gray) curve] and the unstable oscillation
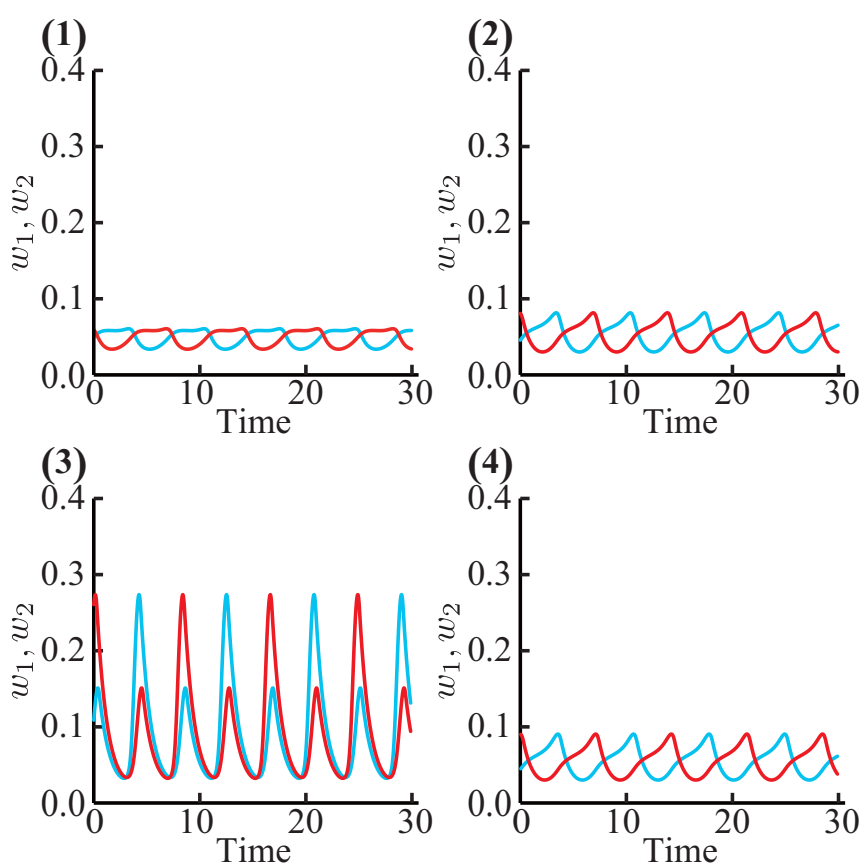

(4)
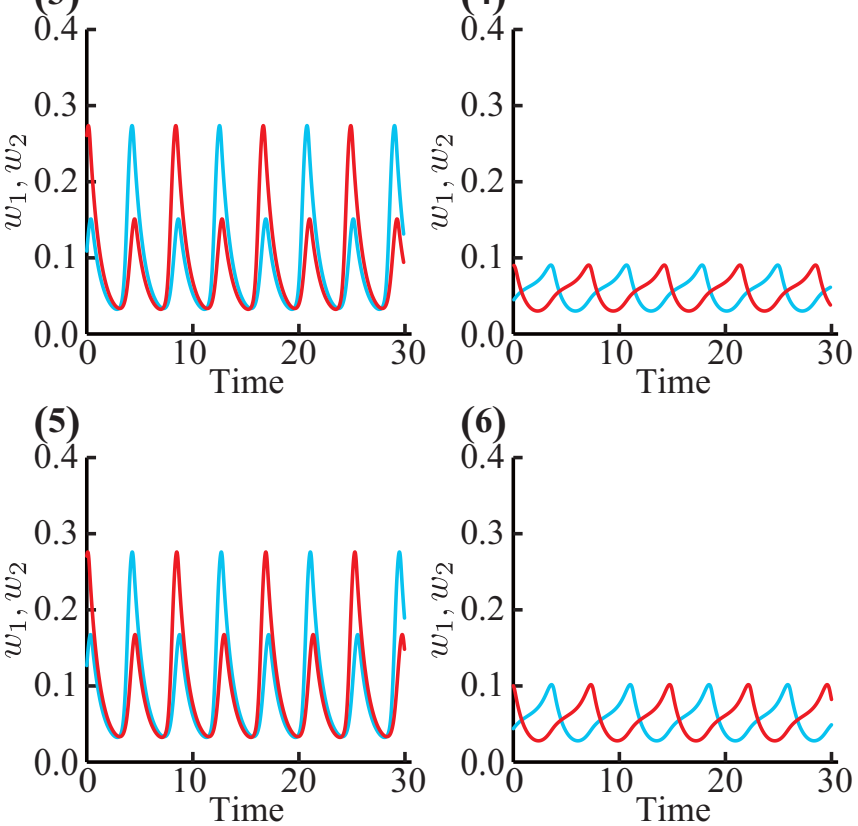

(6)

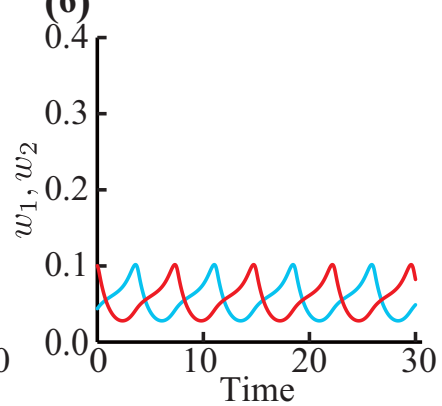

FIG. 4. Time profiles of $w_{i}$ for unstable solutions. These are antiphase oscillations. Parameter $s$ is same value as $s=0.5$. Diffusion parameters are (1) $D=0.3$, (2) $D=0.1$, (3), (4) $D=0.05$, and (5) and (6) $D=0.0$. These profiles correspond to the numbers in bifurcation diagrams, as shown in Fig. 2. We got these profiles by AUTO.

[green (light gray) curve] in [Fig. 2(c-i) and 2(c-ii)]. After the reconnection, the branch of the antiphase oscillation is connected to the period-doubling bifurcation point [Fig. 2(b-i) and 2(b-ii)]. See also Fig. 5 for an illustrative explanation for the reconnection of the bifurcation branches.

It should be noted that there are the oscillations $\left(\mathbf{u}_{1}(t)\right.$, $\left.\mathbf{u}_{2}(t+\theta)\right)$ for an arbitrary $\theta$ at $(s, D)=(0.0,0.0)$. However, only the branches of in-phase and antiphase oscillations continue when we change $s$.

\section{EXPERIMENT}

To confirm the above theoretical results, we performed experiments using the photosensitive $\mathrm{BZ}$ reaction with $\mathrm{Ru}(\mathrm{bpy})_{3}^{2+}$ catalyst. Two BZ solutions were connected by a pump and light feedback system (Fig. 6). More detailed schematic illustrations are as shown in Fig. 7. 


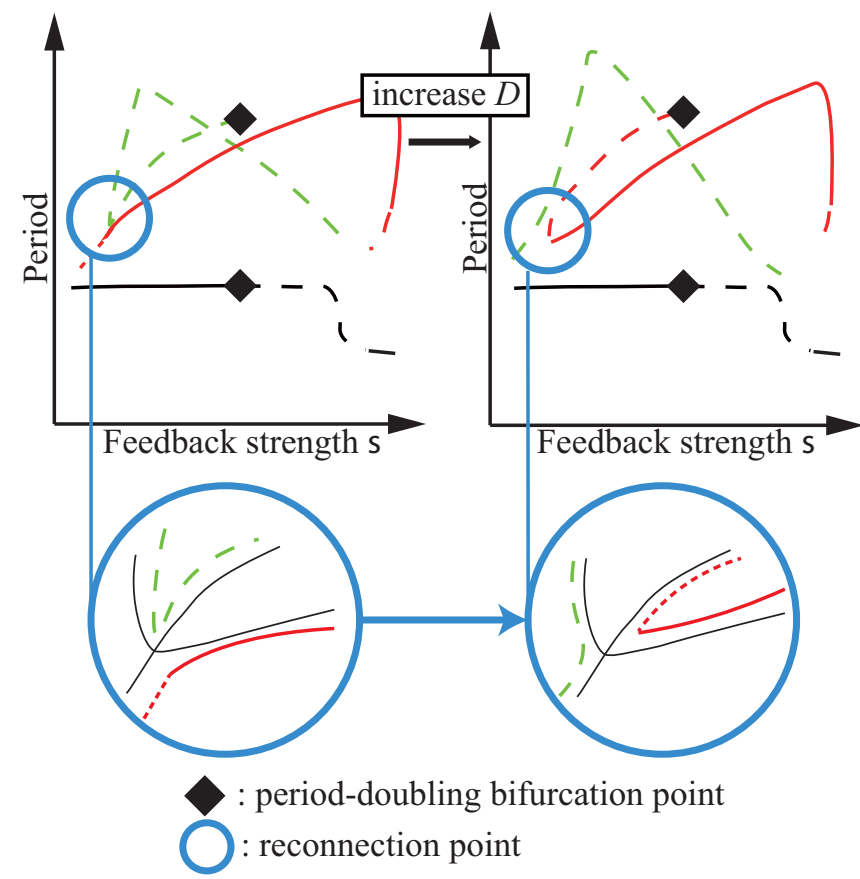

FIG. 5. Schematic diagram for reconnection. Black, red (dark gray), and green (light gray) lines correspond to Fig. 2.

\section{A. Experimental setup}

The experiments were performed using a continuous-flow stirred tank reactor (CSTR) system [see also Fig. 7(a) for the detailed setup]. The following two stock solutions were continuously supplied into the reaction tank; one was $\mathrm{NaBrO}_{3}$, $\mathrm{H}_{2} \mathrm{SO}_{4}, \mathrm{CH}_{2}(\mathrm{COOH})_{2}$, and $\mathrm{Br}^{-}$and the other was $\mathrm{Ru}(\mathrm{bpy})_{3}^{2+}$. Tygon tubes and peristatic pumps (AS-ONE, SMP-23AS) were used to supply and discharge of the solutions. The flow rate of each stock solutions was $9 \mathrm{ml} / \mathrm{h}$, and thus the residence time was $33 \mathrm{~min}$. The volume of a reactor solution was kept to $10 \mathrm{ml}$ by the discharging tube. The final concentrations in the reaction solution were $\left[\mathrm{NaBrO}_{3}\right]=0.150 \mathrm{M}$,

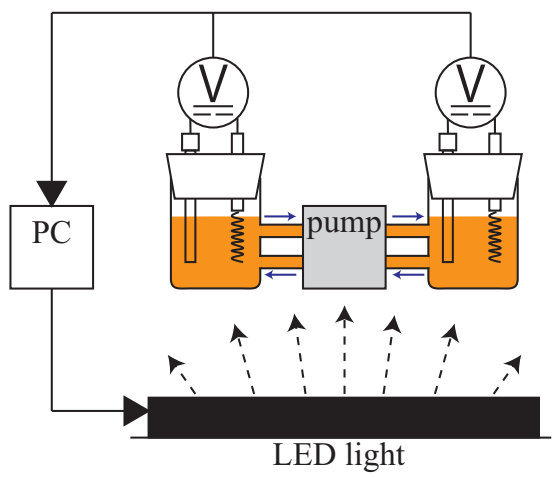

FIG. 6. Schematic illustration of the experimental setup.

$\left[\mathrm{H}_{2} \mathrm{SO}_{4}\right]=0.450 \mathrm{M},\left[\mathrm{CH}_{2}(\mathrm{COOH})_{2}\right]=0.100 \mathrm{M},[\mathrm{NaBr}]=$ $0.012 \mathrm{M}$, and $\left[\mathrm{Ru}(\mathrm{bpy})_{3}^{2+}\right]=0.560 \mathrm{mM}$. For the coupled oscillator system, two reactors were connected by the peristaltic pump system, whose flow rate was $30-507 \mathrm{ml} / \mathrm{h}$. The experiments were performed in an air-conditioned room at $25^{\circ} \mathrm{C}$.

To construct the feedback system, the BZ solutions were illuminated by the same light-emitting diode (LED) whose intensity was determined by their electric potentials. The potential was measured using electrodes ( $\mathrm{Pt}$ wire and $\mathrm{Ag} / \mathrm{AgCl}$ electrode) that were connected to an Arduino. The Arduino system is an analog-digital converter [see also Fig. 7(b) for the detailed setup]. The Arduino got positive electric potential value $V_{i}(t)$ for $\mathrm{BZ}$ solution $i(i=1,2)$. Depending on the input value $V_{i}(t)$, the Arduino outputs positive integer value $I(t)$ which is in the range of 0 to 255 . Let $\psi_{i}(t)$ be a normalized voltage by the Arduino for each BZ solution $i(=1,2)$. To determine the output value $I(t), \psi_{i}(t)$ was calculated as follows:

$$
\psi_{i}(t)=\left[V_{i}(t)-V_{\min , i}\right] \frac{255}{V_{\mathrm{ref}, i}} .
$$

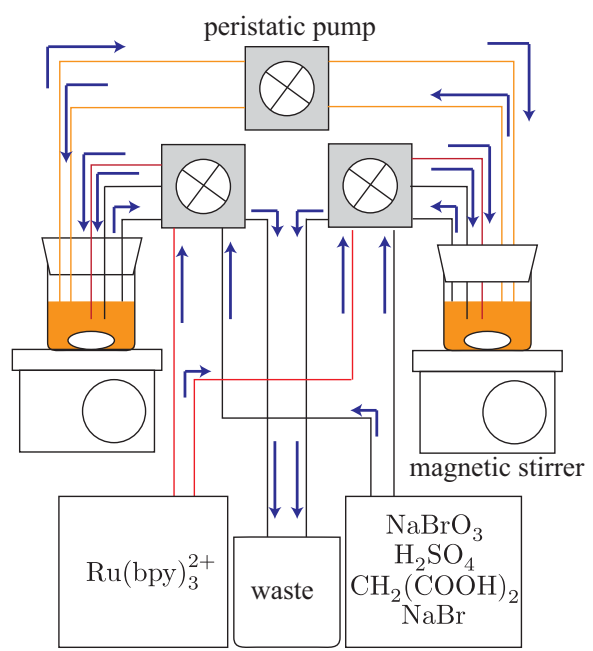

(a)

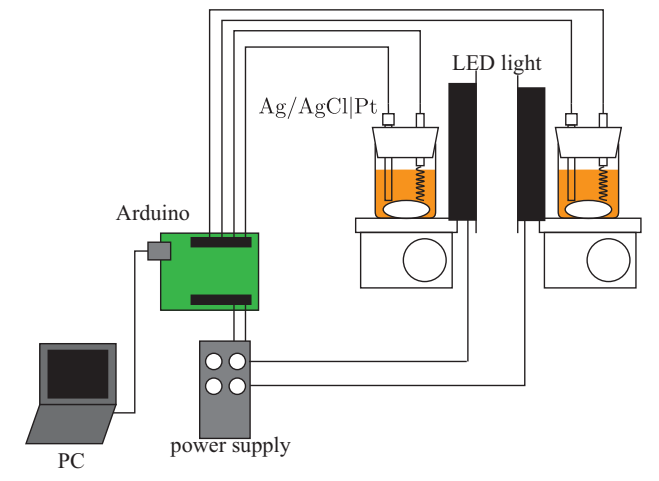

(b)

FIG. 7. Schematic illustarions of the detail experimental setup. (a) CSTR system and (b) light feedback system. 


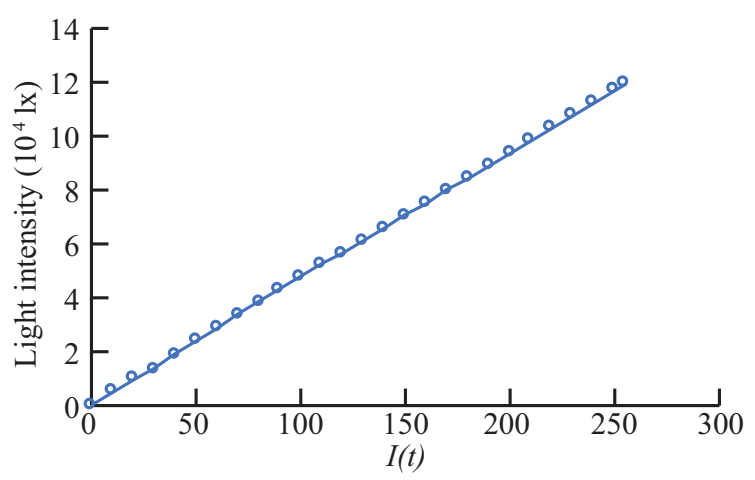
$I(t)$.

FIG. 8. Light intensity of the LED depending on the output value

Here $V_{\min , i}(t)$ is the minimum value for $V_{i}(t)$ and $V_{\text {ref, } i}$ is a positive constant defined as follows:

$$
V_{\mathrm{ref}, i}=V_{\max , i}-V_{\min , i}
$$

Here $V_{\max , i}$ is the maximum value for $V_{i}(t)$. The value of $I(t)$ was determined by $\psi_{i}(t)$ using the following equation:

$$
I(t)=\sigma \times \frac{\psi_{1}(t)+\psi_{2}(t)}{2} .
$$

Here $\sigma(0.1 \sim 0.6)$ denotes the feedback strength parameter. The value of $V$ was recorded in a PC every $100 \mathrm{~ms}$. The Arduino was connected by a control cable (AITEC SYSTEM, model no. TCB3P) to an LED power supply
(AITEC SYSTEM, TPAP2B02430NCW) and it controlled the light intensity of LED lights (AITEC SYSTEM, model no. LMG100X120-22WD-4). The light intensity was determined by the value of $I(t) . I(t)$ between 0 and 255 was calibrated to the illumination intensity in $1 \mathrm{x}$, as shown in Fig. 8.

We performed two experiments with different flow rates (high: $350 \mathrm{ml} / \mathrm{h}$ and low: $150 \mathrm{ml} / \mathrm{h}$ ). In the case of a high flow rate, the value of $\sigma$ was set to 0.1 as the initial condition and the oscillation was measured until the synchronized behavior was maintained for $10 \mathrm{~min}$. After the synchronized behavior reached the stable state, the feedback parameter $\sigma$ was changed to the next value, and the oscillation was observed again until the system reached the next stable state. The feedback parameter $\sigma$ was increased from 0.1 to 0.5 at first and subsequently decreased to 0.2 .

With a low flow rate, the feedback parameter $\sigma$ was changed from 0.6 to 0.2 and the stable synchronized behavior was obtained for each $\sigma$. In addition, we artificially prepared in-phase oscillations as the initial condition, and the synchronized behavior was measured with changing $\sigma$ from 0.2 to 0.4 .

\section{B. Experimental results}

Two oscillators were synchronized typically within several minutes after changing the value of $\sigma$. We define the synchronized behavior as stable when the behavior is maintained for more than $10 \mathrm{~min}$.

We obtained the bifurcation diagrams against parameter $\sigma$ for two different flow rates. In the case of strong diffusion (a)

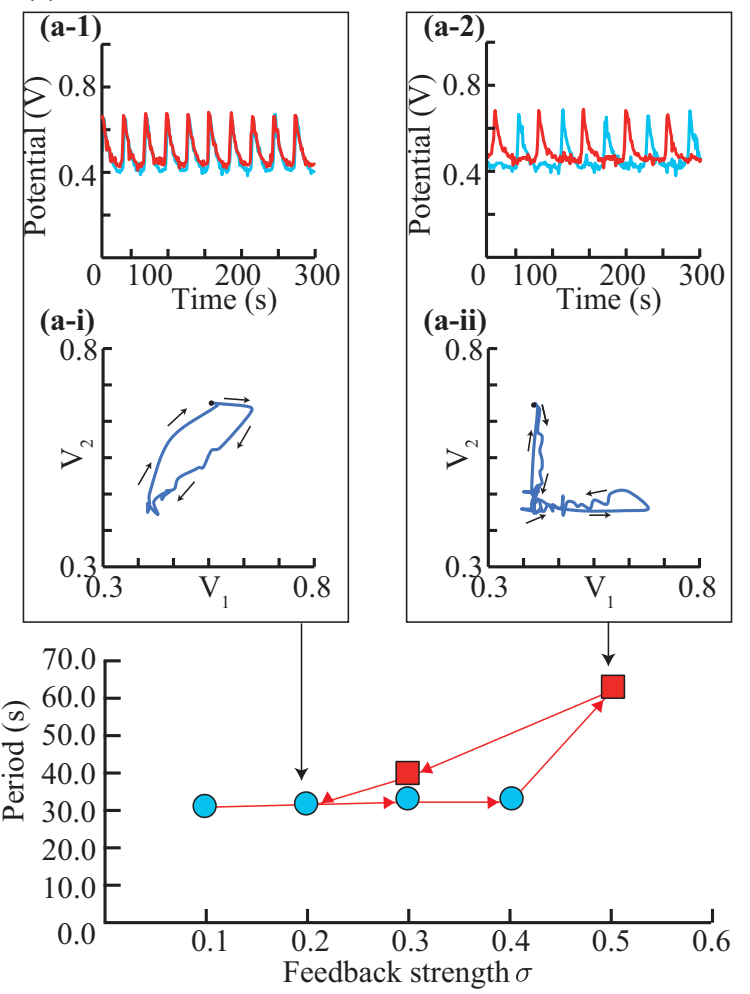

(b)

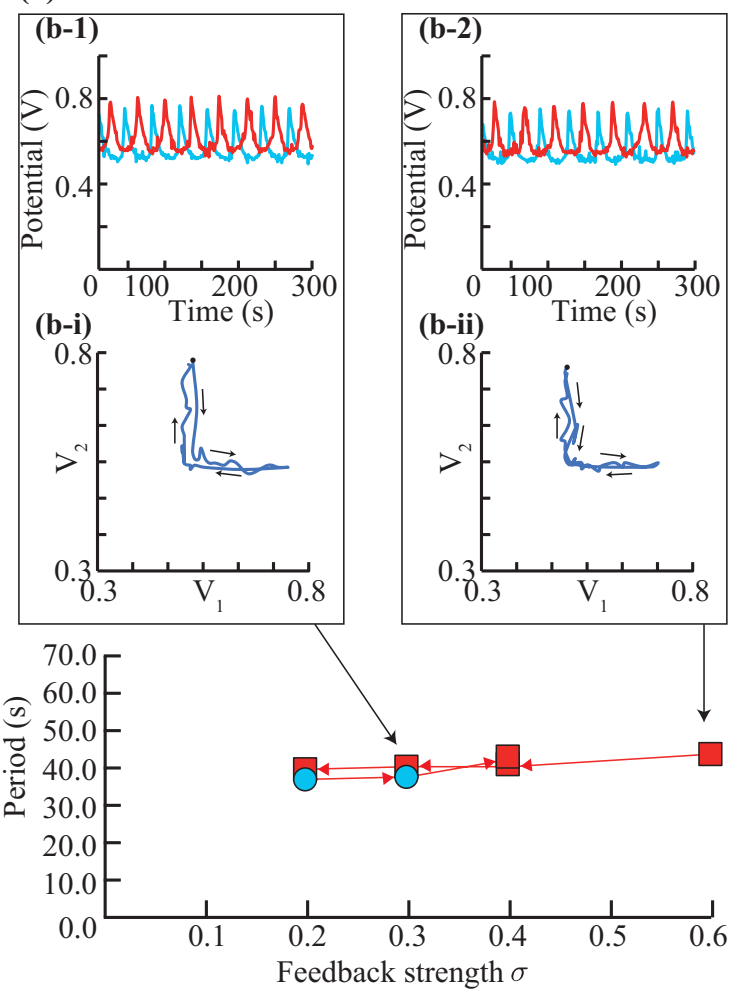

FIG. 9. The bifurcation diagram obtained by the experiments. The period of oscillation was plotted against $\sigma$. Blue (light gray) circles and red (dark gray) squares indicate in-phase and antiphase oscillations, respectively. The flow rate was (a) $350 \mathrm{ml} / \mathrm{h}$ and (b) $150 \mathrm{ml} / \mathrm{h}$. The error bars were sufficiently small to be hidden behind each plot. Insets show time profiles [(1) and (2)] and phase plane plots [(i) and (ii)] of $V_{i}$. 
coupling (flowrate $=350 \mathrm{ml} / \mathrm{h}$ ), in-phase oscillations were initially observed at $\sigma=0.1,0.3$, and 0.4 and transited to antiphase oscillations at $\sigma=0.5$. The period increased from $32.0 \mathrm{~s}$ at $\sigma=0.4$ to $61.2 \mathrm{~s}$ at $\sigma=0.5$ [Figs. 9(a-2) and 9 (a-ii)]. With a decrease in $\sigma=0.3$, the period decreased to $38.6 \mathrm{~s}$, which is an antiphase oscillation. A further decrease in $\sigma$ induced the transition to in-phase oscillation where the period was independent of $\sigma$ [Figs. 9(a-1) and 9(a-i)].

Meanwhile, with weak diffusion coupling (flowrate $=$ $150 \mathrm{ml} / \mathrm{h}$ ), antiphase oscillations were observed at $\sigma=0.6$ [Figs. 9(b-2) and 9(b-ii)]. The antiphase oscillation was stably observed to $\sigma=0.2$ [Figs. 9(b-1) and 9(b-i)]. When the initial condition was set to the in-phase oscillation, the stable synchronized behavior was an in-phase oscillation for $\sigma=0.2$ and 0.3 and was an antiphase oscillation for $\sigma=0.4$.

\section{DISCUSSION}

We discuss based on numerical simulation and experimental results. It should be noted that the electric potential $V_{i}$ is positively related to $w_{i}\left[=\right.$ concentration of $\left.\mathrm{Ru}(\mathrm{bpy})_{3}^{3+}\right]$. Therefore, the mathematical model (2) qualitatively corresponds to the experimental setup. Furthermore, we can consider the comparison between numerical simulation and experiment by the comparison between feedback strength parameter $s$ and $\sigma$.

The experimental result for the strong diffusion effect, as shown in Fig. 9(a), is consistent with the bifurcation diagram, as shown in Fig. 2(a-ii) or Fig. 2(b-ii). This is because, as we have described in the previous section, we observed a transition from in-phase to antiphase oscillation by increasing the feedback strength and a back-transition from antiphase to in-phase oscillation by decreasing the feedback strength. This can be explained by the fact that two stable branches of in-phase and antiphase oscillation are not connected with each other but connected by the unstable branch of the un- stable oscillations. In this case, the transitions of period, i.e., the period of antiphase oscillations became approximately twice that of in-phase oscillations, correspond between the numerical simulation and the experiment. Furthermore, the experimental result for the weak diffusion effect, as shown in Fig. 9(b), corresponds to the bifurcation diagram, as shown in Figs. 2(c-ii) or 2(d-ii). In particular, an antiphase oscillation was observed even in the weak feedback strength both in the mathematical model and the experiment [Fig. 1(III-i) and Fig. 9(b-1), respectively].

These two different responses against the feedback strength can be clearly explained by the reconnection of the bifurcation branches, as described in Sec. IIC. A similar reconnection can also be observed using the FitzHugh-Nagumo model instead of the three-component Oregonator model. Therefore, we expect that the reconnection is a general phenomenon in oscillatory reaction diffusion systems with global feedback.

\section{CONCLUSION}

We studied a feedback system for the photosensitive BZ reaction through both theoretical and experimental studies. Using a mathematical model, we detected the characteristic bifurcation structure by two-parameter search. These simulation results were confirmed through an experiment.

Although we have only studied one system of coupled oscillators, the results obtained in this study are suggestive even for the reaction diffusion system on a 1D or 2D domain. Two-parameter search by not only the feedback strength but also the diffusion effect is necessary to understand a variety of oscillatory patterns.

\section{ACKNOWLEDGMENTS}

This work was partially supported by JSPS KAKENHI Grants No. JP17K05375 and No. JP16KT0023.
[1] M. Cross and P. Hohenberg, Rev. Mod. Phys. 65, 851 (1993).

[2] V. Gáşpár, G. Bazsa, and M. T. Beck, Z. Phys. Chem. (Leipzig) 264, 43 (1983).

[3] H.-J. Krug, L. Pohlmann, and L. Kuhnert, J. Phys. Chem. 94, 4862 (1990).

[4] I. Hanazaki, J. Phys. Chem. 96, 5652 (1992).

[5] E. Mihaliuk, T. Sakurai, F. Chirila, and K. Showalter, Faraday Discussions 120, 383 (2001).

[6] E. Mihaliuk, T. Sakurai, F. Chirila, and K. Showalter, Phys. Rev. E 65, 065602(R) (2002).

[7] T. Sakurai, E. Mihaliuk, F. Chirila, and K. Showalter, Science 296, 2009 (2002).

[8] K. Nishi, S. Suzuki, K. Kayahara, M. Kuze, H. Kitahata, S. Nakata, and Y. Nishiura, Phys. Rev. E 95, 062209 (2017).

[9] V. Petrov, Q. Ouyang, and H. Swinney, Nature 388, 655 (1997).

[10] V. Vanag, L. Yang, M. Dolnik, A. Zhabotinsky, and I. Epstein, Nature 406, 389 (2000).
[11] V. Vanag, A. Zhabotinsky, and I. Epstein, J. Phys. Chem. A 104, 11566 (2000).

[12] I. Proskurkin and V. Vanag, Phys. Chem. Chem. Phys. 20, 16126 (2018).

[13] K. Kashima, T. Ogawa, and T. Sakurai, Automatica 56, 25 (2015).

[14] Y. Umezu, T. Ogawa, and K. Kashima, Trans. Soc. Inst. Cont. Engin. 51, 110 (2015).

[15] M. Yoshimoto, K. Yoshikawa, Y. Mori, and I. Hanazaki, Chem. Phys. Lett. 189, 18 (1992).

[16] K. Miyakawa, T. Okabe, M. Mizoguchi, and F. Sakamoto, J. Chem. Phys. 103, 9621 (1995).

[17] K. Fujii, D. Hayashi, O. Inomoto, and S. Kai, Forma 15, 219 (2000)

[18] I. Stuchl and M. Marek, J. Chem. Phys. 77, 2956 (1982).

[19] K. Nakajima and Y. Sawada, J. Chem. Phys. 72, 2231 (1980).

[20] R. Field and R. Noyes, J. Chem. Phys. 60, 1877 (1974).

[21] J. Tyson and P. Fife, J. Chem. Phys. 73, 2224 (1980).

[22] "AUTO-07p", http://indy.cs.concordia.ca/auto/. 